\title{
Comparative Visualization of Deep Water Asteroid Impacts on Ultra-high-resolution Wall Displays with Seawall
}

\author{
Adhitya Kamakshidasan* José Galaz ${ }^{\dagger}$ Rodrigo Cienfuegos Antoine Rousseau $^{\S} \quad$ Emmanuel Pietrigall \\ Inria, France \\ Inria, Chile \\ CIGIDEN, Chile \\ Inria, France \\ Inria, France
}

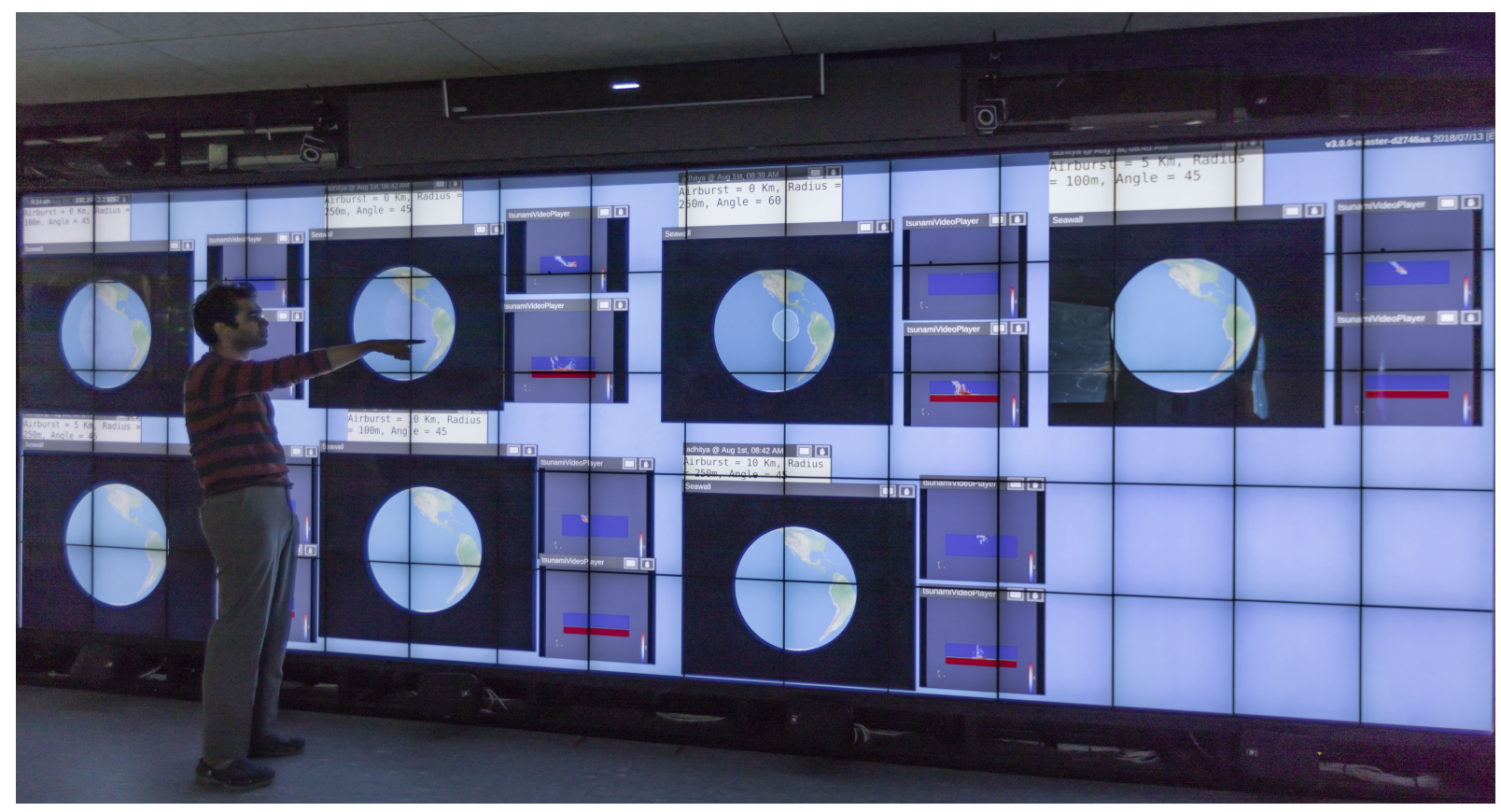

Figure 1: Multiple simulations running simultaneously on the WILDER ultra-wall. The high display capacity of this interactive surface makes it possible to show, for each of the simulations: a planet-wide view showing the propagation of the asteroid-generated tsunami on the globe, a close-up on the region of impact, showing a simulation of one or more scalar fields, parameters of the simulation.

\section{Abstract}

We use a ultra-high-resolution wall display to make it easier for analysts to visually compare and contrast different simulations from a deep water asteroid impact ensemble dataset. Thanks to their very high pixel density, these physically large surfaces enable users to display multiple simulations simultaneously, juxtaposing multiple perspectives on the data for each of them. Playback of the different simulations can be synchronized. Changes of perspective on the data, made interactively in one view by users, can also be applied automatically to the corresponding views in the other simulations.

Index Terms: Human-centered computing-VisualizationScientific visualization; Human-centered computing-Visualization-Geographic visualization

\footnotetext{
*e-mail: adhitya.kamakshidasan@inria.fr

†e-mail: jose.galaz@inria.cl

†e-mail: director@cigiden.cl

$\S$ e-mail: antoine.rousseau@inria.fr

Ile-mail: emmanuel.pietriga@inria.fr
}

\section{INTRODUCTION}

The deep water asteroid impact dataset [7] has two main characteristics that make it difficult to visualize: 1 . it is an ensemble dataset, consisting of multiple simulations of the same phenomenon but with different parameters (airburst, asteroid diameter, and angle of entry); and 2. each simulation is a multi-variate dataset, consisting for the most part of scalar fields (pressure, temperature, velocity along the three axes, etc.) amenable to volumetric rendering or 2D visualization (slicing). It is actually possible to derive additional data from the original dataset, that can be of significant interest to analysts who want to understand the influence of the parameters on the possible generation of a tsunami, and the characteristics of that tsunami.

The problem of visualizing combinations of scalar fields in a given simulation has already been explored by Samsel et al. [12]. Rather than proposing other volumetric renderings for these specific data, we focus here on a complementary question: how to efficiently display multiple simulations in the ensemble simultaneously to facilitate compare \& contrast tasks; and how to display related views on each one of these simulations, to enable analysts to gain additional insights about the influence of the considered parameters.

Displaying multiple simulations simultaneously consumes significant screen real-estate. In previous communications about the study of asteroid-generated tsunamis (AGT), Patchett, Samsel and colleagues typically show up to a maximum of four simulations simultaneously. But displaying multiple complementary views on 


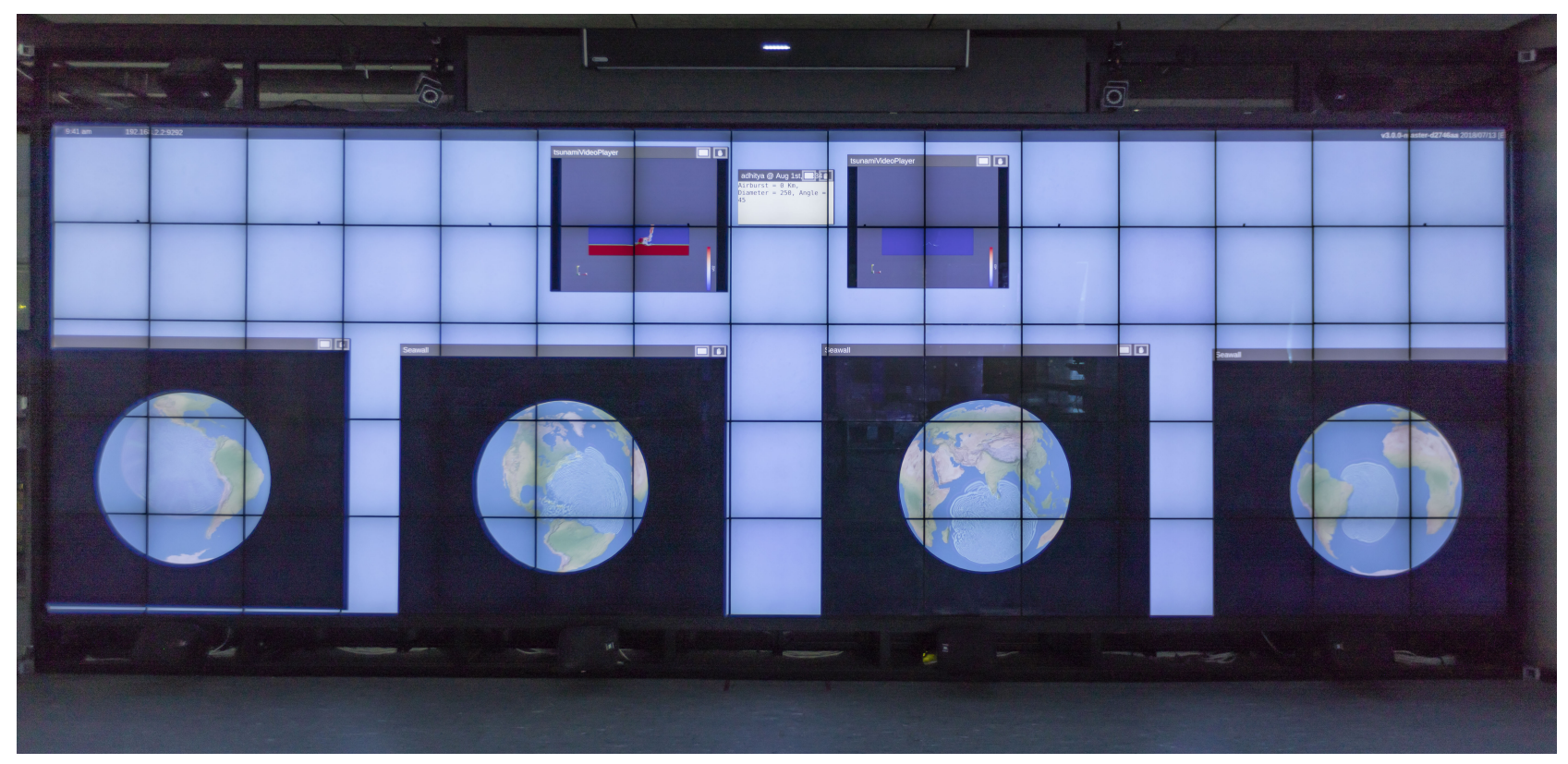

Figure 2: Multiple simulations of the same asteroid (same airburst, size and angle of entry) impacting the Earth in different locations. From left to right: Pacific ocean, Caribbean sea, Indian ocean, South Atlantic ocean.

multiple simulations, all simultaneously, is near impossible on regular workstations. Thanks to their very high pixel density over a large physical surface, cluster-driven ultra-high-resolution wall displays, or ultra-walls for short [5], can accommodate much more data [6]. For instance, the wall display depicted in Figure 1 has a total resolution of $14,400 \times 4,800$ pixels. The other wall in our lab, WILD, has a total resolution of $20,480 \times 6,400$ pixels. Larger walls have appeared since then. Potential applications include, e.g., astronomical data analysis [9], neuroimagery [1] and medical imagery analysis [11], road traffic management [10], air quality monitoring [8] and other geovisualizations. We describe a proof-of-concept visualization environment for the AGT data, called Seawall. The proof-of-concept presented here can display multiple simulations from the deep water asteroid impact dataset, and can show complementary views for each of the simulations on screen, as illustrated in Figure 1.

\section{Seawall}

\subsection{Overview}

Two months ago, we initiated a project to explore the potential of wall displays for the visualization of earthquake-generated tsunamis. The project, called Seawall, is based on an existing Web-based application called TsunamiLab, that we aim at extending to run on cluster-driven ultra-walls. The proof-of-concept we describe here can be seen as an extension to Seawall, that makes the application capable of handling the case of asteroid-generated tsunamis. We thus refer indifferently to the original project and to the proof-of-concept as Seawall. As we are still in the early stages of the overall project, what we present is rather a mockup of what Seawall will eventually be, illustrated on the AGT dataset, as discussed later in this section.

Seawall is implemented on top of SAGE2 [4], the new version of the Scalable Amplified Group Environment written using Web technologies. SAGE2 enables us to run Web applications over the type of hardware configurations that run ultra-walls, typically composed of a cluster of computers driven by a master node [3], hiding much of the complexity associated with such environments. While it is not the most efficient solution in terms of performance and scalability, using SAGE2 enables us to quickly prototype interfaces, and to more easily combine different data sources and visualizations.
Seawall features two visualization modes. In single globe mode, it shows a single visualization, at a very high resolution, of the Earth globe, spanning all screen tiles. In small-multiples mode, it shows multiple globes displaying the different, concurrently running, simulations. The simulations can vary in different ways: different initial conditions, different earthquake/asteroid impact locations on the planet. This second mode is what is of interest to us here.

Showing multiple simulations running concurrently, with control on the playback of each simulation, enables analysts to more easily compare \& contrast the influence of different parameters. Figure 1 shows different simulations of an asteroid impacting the Earth at the same location, but with different parameters (airburst, size, angle of entry). Figure 2 shows different simulations of the same asteroid (i.e., all parameters in the AGT data being equal) impacting the Earth in different locations. These two examples illustrate different compare \& contrast scenarios. In the first case, analysts can assess the influence of variations of the asteroid's parameters, while in the second case, they can evaluate the consequences of the same asteroid impacting the Earth in different locations.

We take advantage of the ultra-wall's high display capacity to show multiple views on each simulation. In the current prototype, we can show: an interactive globe-scale view of the propagation of the asteroid-generated tsunami (if any), and a close-up on the region of impact, showing the original data (any given scalar field) exported from VTK. This could be replaced by one of the more elaborate visualizations described in [12], should they eventually become available. Additional charts useful for data analysis (e.g., relevant time-series) can easily be accommodated on the wall, but were not included in the submission due to a lack of time.

Simulations can be played back in sync or independently. They can also be made to loop. The perspective on all globes can also be synchronized, meaning that any panning, zooming or rotation navigation action made by the user will be automatically forwarded to all globes, as is done with 3D brain scans in [1].

\subsection{Current Limitations}

As mentioned earlier, the Seawall project as a whole is still at a very early stage. It started two months ago, and only one month has been 
spent adapting it to the case of asteroids. AGT data processing (see next section) is not completely streamlined yet and requires some manual intervention. Beyond that, the Seawall front-end is still in its infancy. While we render tsunami simulations in TsunamiLab live using shaders in WebGL on top of Cesium.js when on a regular workstation, we are currently encountering severe performance issues with SAGE2 that prevent us from doing so on the cluster driving our wall display. As a temporary fallback, we are currently pre-rendering the tsunami simulation as a sequence of frames, which then gets applied as a video texture on the Cesium.js Earth 3D globe.

User interaction with the wall display is currently limited to the generic mouse \& pointer SAGE2 interface. Seawall will eventually enable users to interact in a much more direct way with the wall display, using handheld devices [2], mid-air gestures [6], and direct touch.

\section{Asteroid Generated Tsunami Simulation}

Seawall is based on Nami, a WebGL + Javascript library under development at the Pontifical Catholic University of Chile, that aims to ease the development of efficient tsunami simulation based solutions using Web technologies. This library allows to define different kinds of initial conditions and study their results as time series or scalar fields. Nami's simulator calculates the propagation of tsunami waves in a 2D spherical grid, and then extrapolates these values to estimate their impact on the coast using techniques that are common in Tsunami Early Warning Systems (TEWS). Two different approaches are explored to estimate the initial deformation of the water free surface. The first uses detailed volumetric data to infer the deformation at every point in a 2D numerical grid, and the second uses existing formulas that depend only on global parameters of the earthquake or asteroid causing the tsunami.

\subsection{Nami Simulations}

The tsunami simulator consists of a second-order finite difference approximation of the linear Shallow Water Equations (SWE) on the sphere, that uses a staggered grid in space and a leap-frog scheme in time. These equations are an approximation of the Euler equations, and represent the balance of mass and momentum fluxes in a depthaveraged fashion. They can be written as:

$$
\begin{gathered}
\frac{\partial \eta}{\partial t}+\frac{1}{R \cos (\theta)}\left(\frac{\partial M}{\partial \lambda}+\frac{\partial}{\partial \theta}(N \cos \theta)\right)=0 \\
\frac{\partial M}{\partial t}+\frac{g h}{R \cos \theta} \frac{\partial \eta}{\partial \lambda}=0 \\
\frac{\partial N}{\partial t}+\frac{g h}{R} \frac{\partial \eta}{\partial \theta}=0
\end{gathered}
$$

where $\lambda, \theta$ are the longitude and latitude coordinates of the event, and $t$ is the time dimension; $\eta$ and $(M, N)$ are the 2D fields of free surface deformation and horizontal momentum; $h$ is the bathymetry field, and $g=9.81 \mathrm{~m} / \mathrm{s}^{2}$ the gravity acceleration. The numerical grid spans the whole globe, except for the poles to avoid grid singularities. Shore lines are considered as fully reflective internal boundaries.

Two different approaches are explored to extract the initial condition from the dataset. The first one uses the water volume fraction $3 \mathrm{D}$ field ( $\mathrm{v} \otimes 2$ in the dataset) to extract the contour surface $\mathrm{v} \otimes 2=1$ that is then interpolated into a uniform $2 \mathrm{D}$ grid over a subrectangle of the numerical domain. The time frame is chosen according to [13], by selecting the instant when most of the surface moves from a downward to an upward direction. The second approach instead, approximates the initial deformation as a parabolic cavity as:

$$
\eta_{0}(x, y)= \begin{cases}-D_{c}\left(1-r^{2}\right) / R_{C}^{2} & r \leq R_{D} \\ 0 & r>R_{D}\end{cases}
$$

where $(x, y)$ is a point located in a cartesian coordinate system whose center is the same as that of the asteroid cavity, and $r=\sqrt{x^{2}+y^{2}}$; $D_{C}$ is the cavity depth; and $R_{C}$ and $R_{D}$ are the inner and outer cavity radii. $R_{C}, R_{D}$ and $D_{C}$ can simply be obtained by fitting this parabola to the data, but we instead obtain it using a simpler formula, used for instance by [13]:

$$
D_{C}=d_{c} / 3=Q R_{I}^{3 / 4} ; \quad Q=\left(8 \varepsilon V_{I}^{2} / 9\left(\rho_{w} g\right)^{1 / 4}\right.
$$

with $d_{c}=2 R_{C} ; R_{I}, V_{I}$ the asteroid radius and speed; $\varepsilon$ the fraction of energy transferred from the asteroid to the tsunami; and $\rho_{w}$ the water mass density. Though $R_{I}$ is known a priori, $V_{I}$ and $\varepsilon$ are calculated from the datasets by taking the respective values and integrating all contributions of $1 / 2 \rho_{w} g \eta_{0}^{2}$ from the inferred initial surface with the other approach.

Finally, for a given Point Of Interest (POI) located in shallow water with bathymetry $h_{P O I}>0$, we estimate its surface elevation using Green's Law:

$$
\eta_{P O I}=\eta_{d}\left(h_{d} / h_{P O I}\right)^{0.25}
$$

where $\eta_{d}$ is the water elevation at the closest grid point located in deep water with bathymetry $h_{d}$. Though this approach oversimplifies the processes behind tsunami inundation, it has proven sufficient to provide useful values compatible with the orders of magnitude expected by TEWS.

\subsection{Data preprocessing}

We use data from [7] to demonstrate our results. Each asteroid simulation provided in the ensemble dataset has three stages of evolution: approach, impact and aftermath. For each simulation, we are interested in extracting parameters about the impact of the asteroid on the surface of the water. We process all time-steps provided, with the intent of finding the deepest impression of the asteroid on the water surface, as explained in the previous section. To infer parameters of equation (5), we use the average velocity of the asteroid.

\section{REFERENCES}

[1] M. Beaudouin-Lafon, S. Huot, M. Nancel, W. Mackay, E. Pietriga, R. Primet, J. Wagner, O. Chapuis, C. Pillias, J. R. Eagan, T. Gjerlufsen, and $\mathrm{C}$. Klokmose. Multisurface interaction in the wild room. IEEE Computer, 45(4):48-56, 2012. doi: 10.1109/MC.2012.110

[2] O. Chapuis, A. Bezerianos, and S. Frantzeskakis. Smarties: An input system for wall display development. In Proceedings of the SIGCHI Conference on Human Factors in Computing Systems, CHI '14, pp. 2763-2772. ACM, 2014. doi: 10.1145/2556288.2556956

[3] H. Chung, C. Andrews, and C. North. A survey of software frameworks for cluster-based large high-resolution displays. IEEE Transactions on Visualization and Computer Graphics, 20:1158-1177, August 2014. doi: 10.1109/TVCG.2013.272

[4] T. Marrinan, J. Aurisano, A. Nishimoto, K. Bharadwaj, V. Mateevitsi, L. Renambot, L. Long, A. Johnson, and J. Leigh. Sage2: A new approach for data intensive collaboration using scalable resolution shared displays. In 10th IEEE International Conference on Collaborative Computing: Networking, Applications and Worksharing, pp. 177-186, Oct 2014. doi: 10.4108/icst.collaboratecom.2014.257337

[5] M. Nancel, E. Pietriga, O. Chapuis, and M. Beaudouin-Lafon. Midair pointing on ultra-walls. ACM Trans. Comput.-Hum. Interact., 22(5):21:1-21:62, Aug. 2015. doi: 10.1145/2766448

[6] M. Nancel, J. Wagner, E. Pietriga, O. Chapuis, and W. Mackay. Midair pan-and-zoom on wall-sized displays. In Proceedings of the 2011 annual conference on Human factors in computing systems, CHI '11, pp. 177-186. ACM, 2011. doi: 10.1145/1978942.1978969

[7] J. M. Patchett and G. R. Gisler. Deep water impact ensemble data set. Technical Report LA-UR-17-21595, Los Alamos National Laboratory, May 2017. 
[8] E. Pietriga and O. Chapuis. Monitoring Air Quality in Korea's Metropolises on Ultra-High Resolution Wall-Sized Displays. International Workshop on Urban Data Science and Technology at Asia Data Week, Nov. 2017.

[9] E. Pietriga, F. del Campo, A. Ibsen, R. Primet, C. Appert, O. Chapuis, M. Hempel, R. Muñoz, S. Eyheramendy, A. Jordan, and H. Dole. Exploratory visualization of astronomical data on ultra-high-resolution wall displays. In Proceedings of the Astronomical Telescopes and Instrumentation conference: Software and Cyberinfrastructure for Astronomy III, 9913, pp. 0W:1-0W:15. SPIE, June 2016. doi: 10. $1117 / 12.2231191$

[10] A. Prouzeau, A. Bezerianos, and O. Chapuis. Towards road traffic management with forecasting on wall displays. In Proceedings of the 2016 International Conference on Interactive Surfaces and Spaces, ISS '16, pp. 119-128. ACM, 2016. doi: 10.1145/2992154.2992158

[11] R. A. Ruddle, R. G. Thomas, R. Randell, P. Quirke, and D. Treanor. The design and evaluation of interfaces for navigating gigapixel images in digital pathology. ACM Trans. Comput.-Hum. Interact., 23(1):5:1-5:29, Jan. 2016. doi: $10.1145 / 2834117$

[12] F. Samsel, J. M. Patchett, D. H. Rogers, and K. Tsai. Employing color theory to visualize volume-rendered multivariate ensembles of asteroid impact simulations. In Proceedings of the 2017 CHI Conference Extended Abstracts on Human Factors in Computing Systems, CHI EA '17, pp. 1126-1134. ACM, 2017. doi: 10.1145/3027063.3053337

[13] S. N. Ward and E. Asphaug. Asteroid impact tsunami: a probabilistic hazard assessment. Icarus, 145(1):64-78, 2000. doi: 10.1006/icar. 1999.6336 